\title{
SOME ASPECTS OF THE MANAGEMENT OF ORTHOPAEDIC CASES IN SOUTH-EAST ASIA
}

\author{
G. M. MülleR, M.B.(LOND.), F.R.C.S. \\ Orthopadic Consultant, Colombo, Ceylon. Formerly Orthopsdic Consultant, Ministry of Health, England
}

THE countries of South-East Asia are generally blessed with a warm and humid climate though there are areas where temperate conditions prevail and even seasonal bitter cold exists. The majority of the inhabitants do not have to endure the encumbrances of heavy clothing or of stout footwear. This freedom conduces to greater bodily mobility, as is well exemplified by the ease and absolute sureness with which pedestrians barely sway away from passing vehicles with an insouciance one does not find in the more heavily garbed and traffic-conscious Westerner. The increased mobility has at least one immediate and desirable consequence in that the liability to sprains of the ankle and knee is much less than it is among Europeans. It is fair to deduce that the unshod foot, moving more quickly and lightly, does not provide the fixed point which is necessary for the production of these injuries. For the same, reason, tearing of the menisci of the knee is relatively uncommon.

\section{The Effects of Going Barefoot}

The advantages of going barefoot are numerous. The thickened and calloused stratum corneum of the foot acts as a buffer to contusions of the sole and provides the foot with a built-in set of anterior arch supports. The examination of footprints on a wet, sandy beach show clearly the differences between the gaits of habitually barefooted and habitually shod persons. In the former the imprint of the toes is deep and discrete, the result of the powerful action of the flexors and intrinsics of the foot as the toes are made to do their part both in gripping the terrain and in pushing off. In the latter, the footprint appears much less dynamic and the imprint tends to be more even throughout its length. The big toe plays an important part in most of the ordinary avocations of life, both in the domestic sphere where it is used for steadying kitchen utensils and in cottage industries like coir making, weaving, the making of fishermen's nets and cadjan making. The hallux varus so commonly seen (Fig. I) and the mobility of the toes are secondary to the increased function assumed by the bare foot. Hallux valgus and bunion formation are seldom met with in the unshod, and when observed are always symptomless; hammer toes and metatarsalgia are unknown. This habit, then, is responsible for much of the difference between the average operation lists in an Eastern country and a Western, where operations on the toes and on the cartilages of the knee play a regular if not an important part. Amputation of the toes, especially of all the toes at once, often desirable in rheumatoid and pre-gangrenous conditions in the West, is not feasible here and fortunately not necessary.

There are some positive dangers associated with the bare foot. Apart from its vulnerability in crush accidents or in traffic, the bare foot requires a longer and more thorough pre-operative cleansing. It is necessary to keep patients due for foot operations at least a day in bed for this purpose. Unless this is done operative wounds at and below the ankle are liable to be contaminated with organisms of the $B$. coli group, $B$. proteus and $B$. pyocyaneus. Steinman's pins through the lower tibia or through the os calcis are introduced

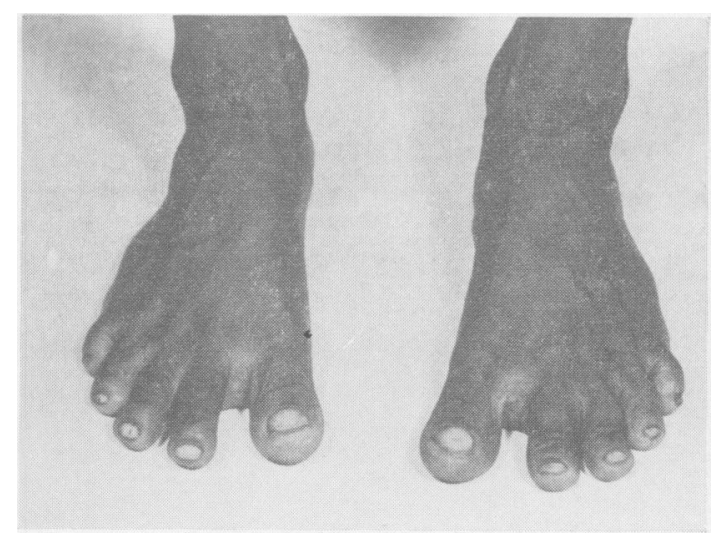

FIG. 1.-Hallux varus. 


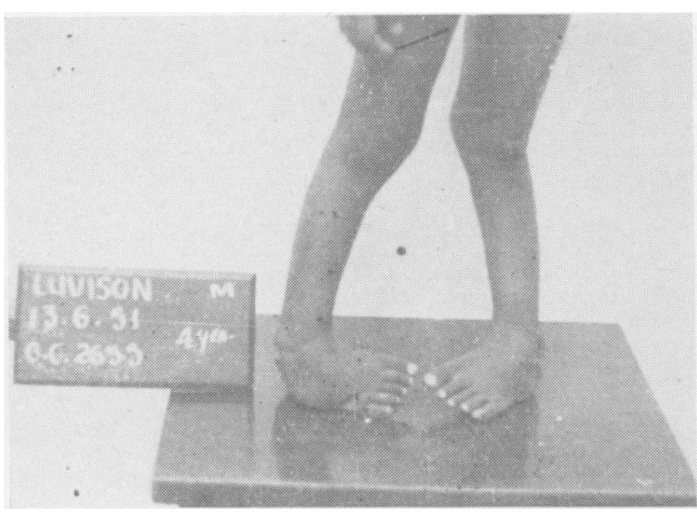

Fig. 2.--A late cases of talipes.

only after a meticulous toilet, and they are left in for as short a time as possible.

An aspect of the barefoot condition as it affects the orthopædic surgeon is the problem of neglected congenital talipes equino-varus. Since the child walks as best he can, his full weight tends to have a further deforming effect on the foot, with the result that numerous cases with extreme deformi-

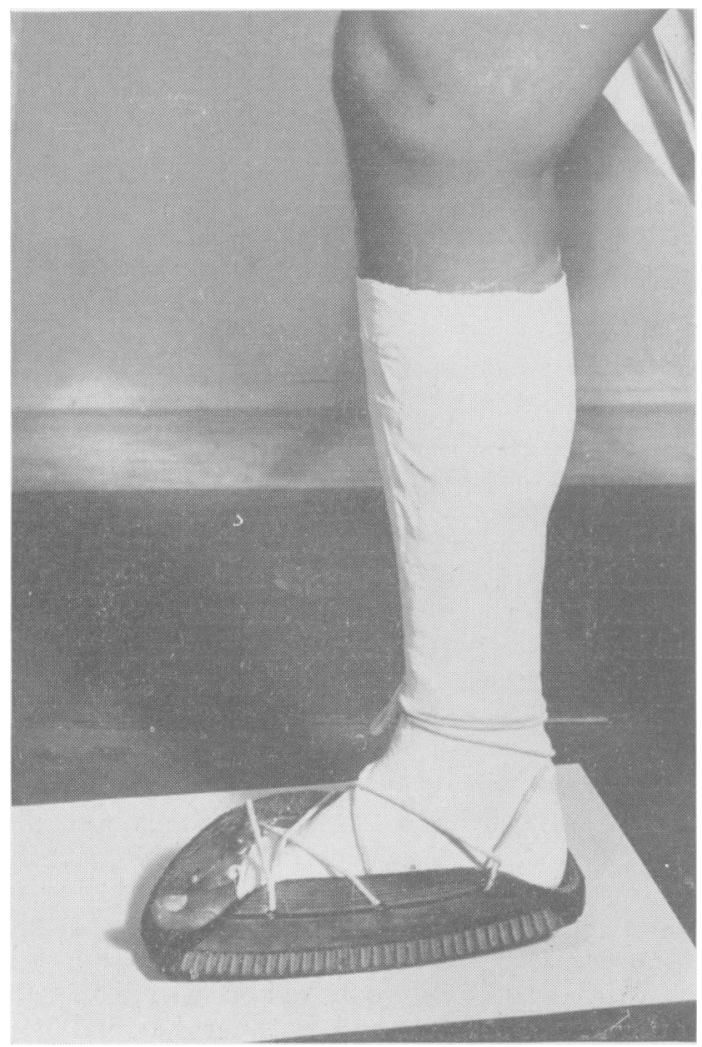

FIG. 3.-Rubber type sole.

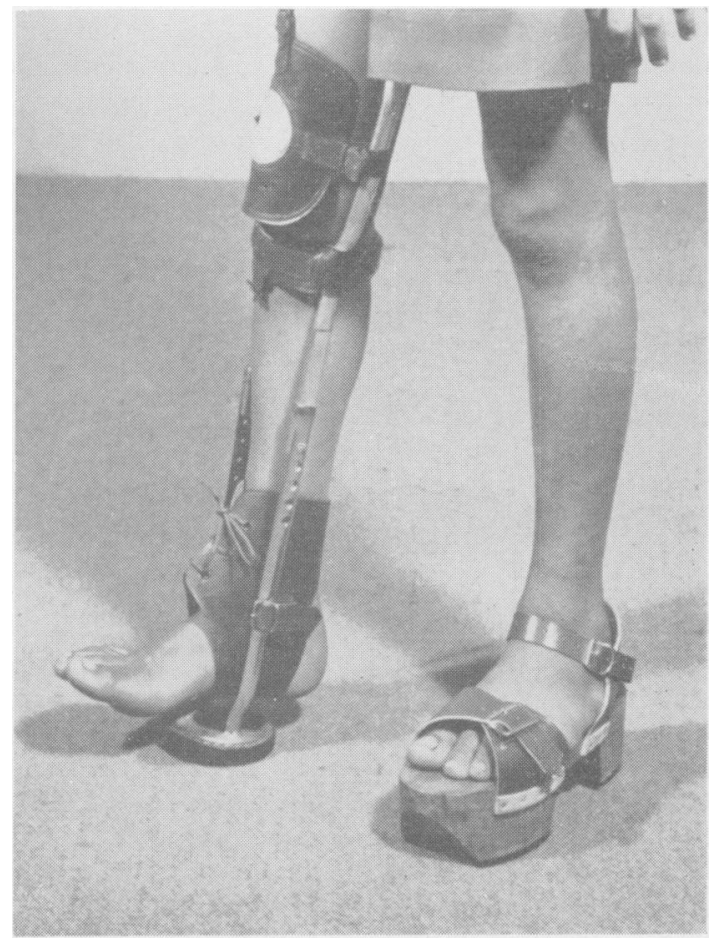

FIG. 4.-Round-bottomed calliper and wooden patten on sound side.

ties in three planes are seen. They are often complicated by the formation over the dorsum of an outsize bursa which has taken the brunt of the pressure against the ground. The correction of this advanced deformity (Fig. 2) requires three stages, viz. correction of equinus with an extensive capsulotomy and perhaps a lengthening of the peroneal and the flexor longus hallucis tendons in the first stage, a wedge tarsectomy in the second stage and a tibialis anticus transplant dorsolaterally in the third stage. After the successful completion of these operations there are few happier sights than that of such a child padding off on plantargrade feet.

Another aspect of barefoot orthopædics is seen in Perthes' disease and in fractures. A simple wooden clog with a broad dorsal leather strap is easily turned out to serve as the patten for the sound side. The plaster covering the injured foot is kept dry underfoot by the use of pareddown segments of motor-car tyres. These are fitted with laces after being cut to size (Fig. 3). In cases of Perthes' disease, a round-bottomed calliper on the affected side does away with the need for wearing a shoe (Fig. 4).

The increased laxity of joints has one drawback as far as the sacro-iliac is considered. This joint 
has been found, following the trauma of falls or of childbirth, to subluxate more easily than in the West. The results of manipulation are excellent.

\section{Amputation Stumps}

The peoples of the south-eastern Asian countries are smaller-boned than the average Westerner. Their height and weight is less, their bulk is smaller and the length of the limbs less. The last factor requires consideration in the fashioning of amputation stumps. The four main stump measurements in Western countries are as follows: above knee 10 to 12 inches below knee $5 \frac{1}{2} \quad$, above elbow 8 ", below elbow 7

These lengths are quite unpractical when applied to the vast majority of these peoples, particularly females, since the stumps would end at or very near the level of the corresponding joint on the normal side. The addition of the working part (i.e. the part of the prosthesis housing the joint mechanism) would bring the corresponding joint to an asymmetrical level and make the limb longer than that of the opposite side. For instance, a $5 \frac{1}{2}$-in. below-knee stump in an average Asian woman would bring the end of the stump only a little above the level of the ankle joint; reamputation would be necessary in order to make the stump workable. In above-knee stumps a IO- to 12-in. length of bone would in most cases cause the artificial knee joint to project beyond the sound knee when the subject sits down. Further, a poor gait would result from the asymmetry of the knees.

The length of the working part being the important factor, for practical purposes it is best to calculate the length of the above-knee stump from the level of the knee itself. It has been found by trial and error that 4 to 5 in. below the knee joint and 4 to 5 in. above the knee joint make suitable lengths for amputation stumps in SouthEast Asia. As regards forearm stumps and prostheses, views have been influenced by the recent ability of artificial-limb makers to produce a prosthesis that fits long forearm stumps. This prosthesis maintains stability though its socket is short and shallow. In the East, where clothes may not cover prostheses, the amputee cherishes a long stump to his forearm and is able to use it in ways that a prosthesis, however efficient, cannot emulate. For above-elbow amputees a bone length of 8 in. as advocated in the West is much too long. A minimum space of $3 \frac{1}{2}$ in. is required for the fitting of an artificial elbow and any amputation should therefore remove at least $3 \frac{1}{2}$ in. of bone (Müller, 1956).

Syme's amputation is permissible in these

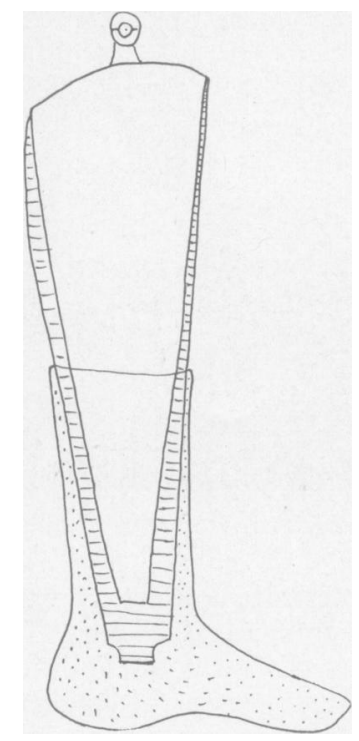

FIG. 5.-Diagram of the prosthesis for rural workers.

climates. The vascularity of the skin is better than in more northern climates and skin flaps tend to be longer-lived and tougher. (This reasoning does not apply to cases of thromboangeitis obliterans, or to diabetic or senile gangrene.) In the barefooted the distal end of a Syme amputation is nearer the floor than in a conventionally clad Westerner, and the owner can stump out on it even without the stimulus of an emergency.

For the usual run of below-knee amputees working in rural conditions a new cheap prosthesis has been devised, consisting basically of a vulcanized-rubber shoe continued sock-wise over a peg-shaped wooden core (Fig. 5). It enables the wearer to walk about in moderately deep paddies and does not have the disadvantage of metal parts which rust early in the tropics and are difficult to replace. The foot of the prosthesis has no heel and is slightly smaller than its normal counterpart; it may easily be worn with an ordinary shoe which has its toe blocked.

\section{Orthopædic Appliances}

One result of the freedom of the limbs normally enjoyed in these climates is the sense of constriction and encumbrance that follows the fitting of any restrictive apparatus. Plaster jackets and casts, spinal braces, callipers, irons and surgical footwear of all kinds are looked on with disfavour; in the case of callipers and footwear the good foot, too, requires to be accoutred in a boot or shoewhich adds to the patient's reluctance. The 
warmth engendered inside plasters and splints is one reason for making the wearing of these obligatory only for a minimum period, and for making them as light as possible.

Another reason for lightness of construction in the case of appliances for a child in South-East Asia is that the latter lacks the robustness found among children in the West; walking is inhibited because of the very weight of the iron or calliper. This is especially so in cases of poliomyelitis affecting the lower extremity where the margin of available power is slender.

The skin under plasters tends to break down more often than in the West. This occurs where the skin is softest, i.e. the arm below the axilla and the inner surface of the thigh. Clearly it is connected with the proliferation of bacteria in these warm and moist regions.

Casts made out of plaster impregnated with resin are quite unpractical in these climates. Plaster, like leather, requires to be porous; otherwise the accumulation of sweat gives rise to sogginess and maceration of the skin, with the danger of ulceration. For this reason resinimpregnated plaster bandages are contraindicated except perhaps for back slats or in making the saddle area of a plaster bed. Plasters also tend to be the natural hiding places for vermin, and since these creatures proliferate enormously in the tropics the need for the greater care of a plaster and its more frequent change is apparent. Occasionally the insufflation of an insecticide inside a plaster has been found of use.

\section{Effects of Climate, Social Habits and Physique}

The warm, humid climate is excellent for certain therapeutic purposes, chiefly the rehabilitation of muscle and joint conditions. Perhaps its most spectacular effect is in the recovery of injured hands, where the greatly increased blood supply is one of the more remarkable differences in vascularity that occur in the tropics, bringing about quicker healing of injuries. A sequel to the enhanced vascularity is the moist and constantly lubricated condition of the skin of the hands and fingers which facilitates rehabilitation. Unfortunately, set off against this physical boon is the innate lethargy of the majority of patients for whom time means very little and to whom the rigours of under-employment are tempered by the solicitude of numerous relations. In the towns there is one added factor which is worth mentioning; compensation is still a little-understood function, and any injured workman likes to believe that a large vista of easy money lies ahead of him. Realization, when it comes, is always something of a shock.

The treatment of the large joints of the lower limb is intimately connected with the personal habits of the people of these countries. Squatting at stool is the common custom and urination even among males is carried out in a squatting position. In immemorial fashion, at rest and in social converse, village people prefer to squat on their heels. A stiff hip or knee joint is therefore an incapacity even less tolerable than in the West. An arthrodesis of the hip, the knee and the ankle should not be done except in the direst necessity. It is much better to accept a mildly osteoarthritic hip involving perhaps some stiffness and pain than to arthrodese such a joint. Even a few degrees of movement in a moderately painful hip are sufficient, with the aid of a mobile lumbar spine, to enable a person to assume a squatting posture. For the same reason it is also advisable to perform a synovectomy or local excision of bone in a knee affected by tuberculosis rather than to stabilize the joint. The availability of the modern antituberculous drugs makes such modified intervention both feasible and successful.

Osteoarthritis, incidentally, especially of the hip, is not anything like as prevalent as in the West, where it is probably the middle-aged patient's greatest scourge. Orthopædic and physiotherapy clinics are thereby saved an immense amount of time and labour. Moreover, the entire gamut of operations devised for the condition over the years and which result in varying degrees of success have only a very small place in the techniques of an orthopædic surgeon here. The saving of time and expensive appliances is automatic and a great boon.

The small size of the bones has some curious sequelæ. The neck of the femur, especially in women, tends to be quite narrow. When a fracture occurs here, greater accuracy, both in the reduction of the fracture and in the introduction of a guide wire, is necessary. For the same reason the low pin operation for pertrochanteric fractures is a much more difficult business and has largely been superseded by pin-and-plate fixation.

The small bulk of muscle in the thigh may be the reason for a type of injury met with here more commonly than in the West-the pertrochanteric fracture in which the main fragments have come apart completely so that on attempted reduction by traction the lower fragment sags an inch or two below the upper and has to be lifted bodily and forcibly into position before the guide wire is passed. Any delay in treating this injury allows callus in quantity to form and renders accurate reduction increasingly laborious.

Kirschner wires of stainless steel are used for the treatment by intramedullary splintage of certain fractures of the radius and ulna. When these wires are difficult to obtain, no ill-effects have been 
noticed to follow the use of ordinary bicycle spokes. It has been my custom to introduce the radial wire through the styloid process just to the dorsal side of the anatomical snuif box; about half-an-inch is left projecting beyond the bone, and the skin is then pulled over it so that the end becomes subcutancous. In the case of the ulna the retrograde method is used, the sharp end of the pin passing down the bone and boring through the lower end. The pin is then removed, reversed and passed through the distal fragment; the lower end is made to emerge through the skin and is drawn further distally before being threaded into the reduced proximal fragment where it embeds itself in the cancellous bone of the olecranon. Any excess over half-an-inch beyond the skin is cut off and the skin drawn over the end. It is not advisable to allow metal to project beyond the skin because of the great likelihood of infection by skin organisms. The leaving of metal in bone is also neither wise nor necessary; screws, plates and wires are generally removed within six months of insertion.

For minor conditions of the hand, owing to the liability to softening from sweating, plaster splints are not as useful as metal ones which are more hygienic and durable. The simple cork-and-wood spatula splint described by Mason (1954) has been found most useful for mallet finger. In Perthes' disease, slinging the leg from the neck (Snyder, 1947) has been found useful and obviates the use of even the simplest calliper (Fig. 6). In cases of unilateral C.D.H. sufficient preliminary traction to bring the head down to the level of the acetabulum has been obtained within two weeks by suspending the affected leg from a Balkan beam with the buttock just off the bed. For small children with drop foot resulting from poliomyelitis, a simple footspring has been made by joining canvas bands round the foot and below the knee with an elastic strap. Village parents are able to look after this and to replace it when necessary, without the need of coming to town for replacement as happens when the regulation toe-spring gives way.

Cases of fracture dislocation of the cervical spine have been treated effectively by using malar hooks attached to a head traction spreader which is connected to weights over the head-rail of the bed. The patient is propped up on a bed rest. The insertion of these malar hooks has rendered the reduction, radiology and treatment of these fractures and fracture dislocations much simpler and more effective.

The location of sinuses, particularly in the hip region, has been made somewhat easier by the device of mixing a radio-opaque dye with methylene blue and injecting the mixture into the sinus. A radiograph then shows the ramifications of the

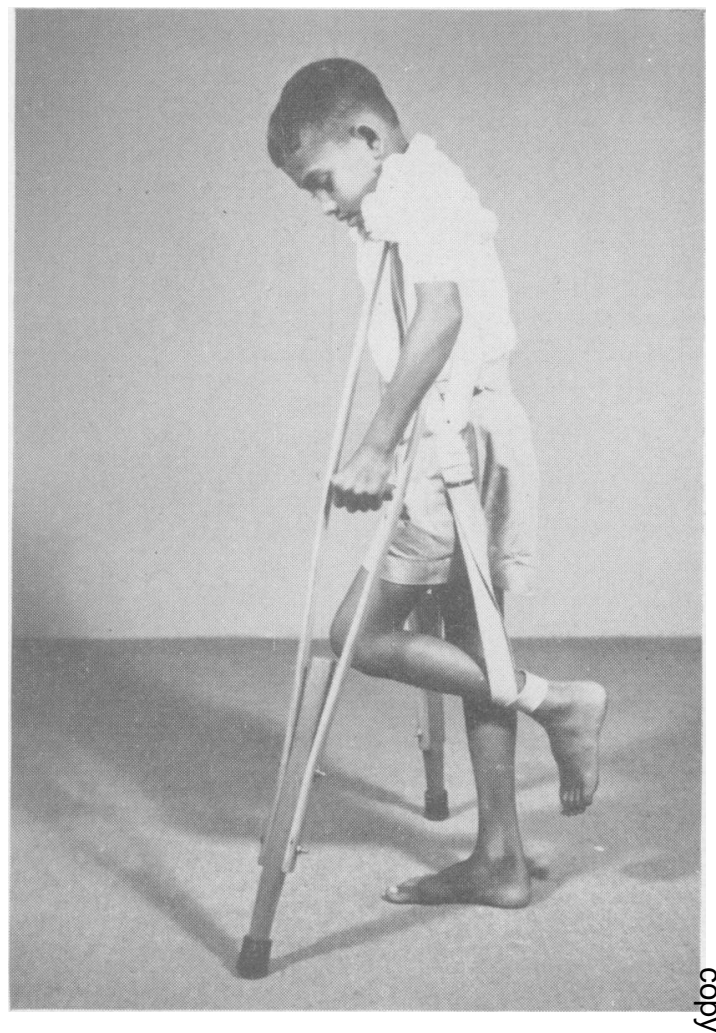

FIG. 6.- Snyder sling for Perthes' disease.

sinus, and if exploration or excision is decided on the colouring matter fixed in the walls of the sinus serves as a guide.

Worthy of note is the rapid healing that occurs in fractures of the tibia; why this should be so is difficult to say, but non-union of the tibia following conservative treatment is particularly uncommon - which is a refreshing change from the West.

The rapid healing of tuberculous affections of bone and joint is also in startling contrast to what happens in Britain where in pre-streptomycin days tuberculous spines seldom healed in less than a year or 18 months. In these countries the immobilization of hospital beds by tuberculous spines is much less of a problem because the healing time for such spines is generally four to six months. A few initial weeks in hospital improve the general condition and help overcome the toxæmia. After that, little harm follows the discharge of the patient in a plaster jacket to home conditions. This method has been found much more satisfactory than prolonged hospitalization. The cause of the rapid response of T.B. bones and joints is not known, but I suspect that it is due to the greatly increased tissue metabolism which occurs in warm climates. 


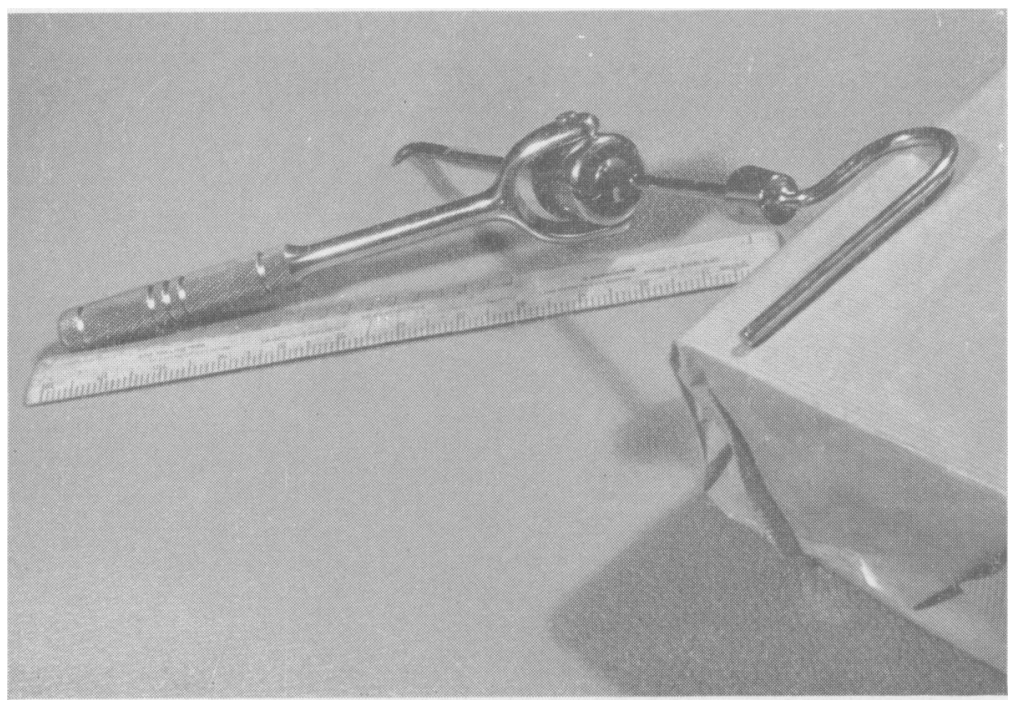

FIG. 7.-Author's extractor for Kuntscher nai's.

Falling off jak or coconut trees or from the rope connecting adjacent trees which tappers of toddy use to cross from one palm to another is an occupational hazard. A fall from these considerable heights, when not otherwise fatal, often involves fractures of the femur and spine. The treatment of suitable fractures of the femur with Kuntscher nails has been a conspicuous success. The nail is introduced by the retrograde method. For removal, the long extractor commonly used was found to be difficult to manipulate because of the limited space between the hip and the axillary region. In the last few years a simpler method of removal has been employed using a hammer head with a keyhole cut, through which is threaded a Io-in. long extractor hook. The hammer head can swivel round a central axis. With this instrument (Fig. 7) extraction of a Kuntscher nail can be carried out in a few minutes under a local anæsthetic.

Colles fractures tend more often to be comminuted with at least one $\mathrm{T}$-shaped split into the wrist joint. It has been disappointing to observe the displacement that has taken place after reduction in these fragments when the usual belowelbow plaster cylinder is applied. Any signs of comminution are now looked on as indicating that more fixation is required. After reduction, therefore, an above-elbow plaster, with the arm at right angles, is applied and kept on for a period of two to three weeks before being changed for a below-elbow one.

One type of injury common here calls for comment-the uncomplicated dislocation of the elbow joint. For some reason the village ayurvedic practitioner seems to be unable to diagnose the dislocation or to deal adequately with it after diagnosis. The joint becomes surrounded by a mass of new bone and fibrous tissue which allows only a few degrees of movement. If the right side is involved the patient is most distressed by his inability to use the hand to feed himself, the left being taboo. The crippling results of this maltreated injury constitute the largest group in the deformities following trauma. Unfortunately the amount of function restored to the joint by an open reduction carried out months or years after the injury only seldom justifies what turns out to be quite a formidable operation.

Myelomatosis, a mysterious disease in any climate, has showed certain facets which may yield rich dividends from further investigation. The disease has been found here much more commonly than in Britain; further, the course of the disease seems to be milder and perhaps on this account to be much more prolonged. The histological types do not show any marked variation from the known pattern; clearly there is some factor, perhaps to do with metabolism, which is responsible for the differences in local behaviour of the disease and which would be most interesting to elicit.

\section{Cerebral Palsy and Poliomyelitis}

The treatment of cerebral palsy, which is a challenge to orthopædic surgeons throughout the world, suffers in these countries from a dearth of ancillary personnel, namely, physiotherapists and occupational therapists. When, therefore, an operation to relieve a certain defect or deformity is feasible no hesitation has been felt in carrying it out; though one is aware that in more advanced 
communities in the West a prolonged course of conservative treatment may have been tried in the first instance. I refer particularly to deformities of the wrist, knee and ankle. In the wrist it has been found that arthrodesis of the joint, by bringing the wrist up from the acutely palmar-flexed position, immediately removes some of the spasticity from the biceps muscle and a much more natural carriage of the limb results. In the lower limb, transposing the hamstring attachments from the tibia and fibula into the femoral condyles has improved stance and gait considerably. Arthrodeses of the Lambrinudi type for foot-drop and elongations of the tendo-Achillis for an equinus deformity are commonly done without waiting to see the effect of prolonged splintage and physiotherapy. A combination of obturator neurectomy carried out through an extra-peritoneal approach and of adductor tenotomy in the thigh serves to alleviate the worst features of the scissor deformity.

There are certain points in the treatment of poliomyelitis in these countries which are worth mentioning. In the majority of cases the first reaction of parents and relations to any evidence of paralysis is to embark on a programme of rigorous rubbing with ointments, some of which have a traditional local reputation for curing paralysis. It has been my impression that the cases showing the most widespread muscle paralyses have been those which have been treated by this method in the first few days following the acute infection. Even in cases seen early it has been difficult to persuade the anxious relations that the best course to follow is one of relative therapeutic inactivity. Thereafter, the major deformities one has to guard against are contractions at the hip, knee and ankle which are encouraged by the ingrained habit of sitting in various postures on the floor during most of the daylight hours. This attitude is, in fact, provocative of a group of deformities, the most striking of which is an abduction-flexion condition of the hip which makes it impossible to fit a walking calliper unless it is corrected. Uncorrected, it causes a marked lumbar lordosis which fortunately is seldom structural in character. Fixed flexion of the knee is another sequel to this habit; when greater than 5 degrees it prevents the fitting of a calliper and requires correction by erasion of the gastrocnemius heads, lengthening of the biceps and semimembranous tendons and often a posterior capsulotomy of the knee. The equinus deformity at the ankle constitutes the third of this triad of deformities which are so typical of untreated poliomyelitis in the East.

\section{Acknowledgments}

I am grateful to Mr. L. P. J. Dias for the loan of some of the apparatus and to Mr. J. Perera for most of the photographs.

\section{REFERENCES}

Mason, M. L. (I 954): Mallet Finger, Lancet i, I 220.

Müller, G. M. (1956): Amputation Stumps, Ceylon med. $\mathcal{F}$., 3 (new series), 2 I 7.

SNyder, C. H. (r947): A Sling for Use in Legg-Perthes' Disease, f. Bone ft Surg., 29, 524 (old series, 45). 